\title{
Emotiv Epoc ile Durağan Hal Görsel Uyarılmış Potansiyel Temelli Beyin Bilgisayar Arayüzü Uygulaması
}

\author{
Abdullah Talha SÖZER ${ }^{* 1}$, Can Bülent FIDAN ${ }^{2}$ \\ ${ }^{1}$ Karabük Üniversitesi, Elektrik-Elektronik Mühendisliği, Karabük \\ ${ }^{2}$ Karabük Üniversitesi, Mekatronik Mühendisliği, Karabük
}

\begin{abstract}
$\ddot{O} \mathbf{z}$
Beyin Bilgisayar Arayüzü (BBA), geleneksel iletişim yöntemlerinin kullanılmasını engelleyen sinir-kas hastalıklarına sahip olan bireyler için yeni bir iletişim seçeneği sunmaktadır. Durağan hal görsel uyarılmış potansiyel (DHGUP) temelli BBA sistemleri sağladığı yüksek hız ve kullanım kolaylığı sebebi ile dikkatleri üzerine çekmektedir. Bu çalışmada Emotiv Epoc elektroansefalografi (EEG) cihazı kullanılarak DHGUP temelli BBA uygulaması gerçekleştirilmiştir. Çalışma, 5 kullanıcının katılımı ile ön hazırlık ve gerçek zamanlı deneyler olmak üzere iki adımdan oluşmaktadır. Ön hazırlık deneyleri ile gerçek zamanlı BBA sisteminde kullanılacak DHGUP tespit metodu ve EEG sinyali toplama süresinin belirlenmesi istenmiştir. Ön hazırlıkta farklı frekansa sahip 12 adet görsel uyaran kullanıcıya sira ile sunulmakta ve 5 saniye boyunca sinyal kaydı yapılmaktadır. Ön hazırlık sinyallerinde 2 saniyelik EEG pencerelerinde \%82.2 DHGUP tespit doğruluğu ve $68.8 \mathrm{bit} / \mathrm{sn}$. bilgi aktarım hızına ulaşılmışırı. Gerçek zamanlı BBA sisteminde ise tuş takımı biçiminde tasarlanan görsel uyaran düzeneği ile kullanıcıların yalnız beyin sinyalleri ile telefon numaralarını yazmalarına imkân sağlanmıştır. Sistem 2 sn. seçim süresi ve $0.5 \mathrm{sn}$. tespit ve geri besleme süresi olmak üzere 2.5 saniyede 1 karakterin yazımına imkan sağlamaktadır. Deneylerde tasarlanan BBA ile 11 haneli telefon numarasının ortalama 40 saniyede yazdırılabildiği gösterilmiştir. Ayrıca çalışmada yüksek DHGUP tespit doğruluğu sağlayan eğitim verisi destekli bir yöntemin Emotiv Epoc ile uygulanabilirliği incelenmiştir. Yöntem, yaygın yöntemlere göre daha yüksek DHGUP tespiti sağlamamıştır. Bu makale DHGUP temelli BBA uygulaması için kılavuz niteliğini taşımaktadır.
\end{abstract}

Anahtar kelimeler: EEG, Beyin Bilgisayar Arayüzü, Durağan Hal Görsel Uyarılmış Potansiyel, Emotiv Epoc.

\section{Implementation of Steady State Visual Evoked Potential based Brain Computer Interface with Emotiv EPOC}

\begin{abstract}
Brain computer interface (BCI) offers a new communication pathway to individuals with neuromuscular disorders that prevent the use of traditional communication channels. The steady state visual evoked potential (SSVEP) based BCI systems take attention since it has high speed and ease of use. In this study, SSVEP based BCI implementation was performed by using Emotiv Epoc electroencephalography (EEG) device. This study consists of two steps, preliminary preparation and real-time experiments with participation of 5 subjects. In preliminary preparation stage, the SSVEP detection method and the EEG signal length that will be used in the real-time BBA were wanted to be decide. In the preliminary preparation stage, 12 visual stimuli with different frequencies are presented to the user in sequence and signal recording is performed for 5 seconds. In preparation signals, $82.2 \%$ SSVEP detection accuracy and $68.8 \mathrm{bits} / \mathrm{sec}$. information transfer rate were reached in the 2 -second EEG epochs. In the real-time BCI, the visual stimuli designed in the form of a keypad allows users to write phone numbers only with the brain signals. The system allows entering a character in 2.5 seconds, including 2 sec selection time and $0.5 \mathrm{sec}$ detection and feedback time. Experiments with 5 users showed that 11-digit phone number can be entered about 40 seconds with the BCI. In addition, it was investigated the applicability of a training data supported method which provides high SSVEP detection accuracy using Emotiv Epoc. The method did not provide higher SSVEP detection accuracy than traditional methods. This article is a guideline for SSVEP-based BCI application.
\end{abstract}

Keywords: EEG, Brain Computer Interface, Steady State Visual Evoked Potential, Emotiv Epoc.

"Sorumlu yazar: talhasozer@karabuk.edu.tr

Geliş Tarihi: 18.07.2018, Kabul Tarihi:14.11.2018 


\section{Giriş}

Son yıllarda odaklanılan araştırma alanlarından biri olan beyin bilgisayar arayüzleri, çeşitli sinir - kas hastalıkları sebebi ile bazı vücut fonksiyonlarını kaybeden kişilere, bu fonksiyonların kısmen de olsa geri kazanılmasına imkân sağlayan yeni bir teknolojidir. BBA, Amyotrophic Lateral Sclerosis (ALS) gibi hastalıklar sebebi ile kaslarının kontrolünü kaybeden bireylere yalnızca beyin sinyallerini kullanarak haberleşme imkânı sunmaktadır [1-4].

Manyetoensefalografi (MEG), Fonksiyonel Manyetik Rezonans Görüntüleme (fMRI) gibi görüntüleme yöntemleri ile beyin sinyalleri toplanabilmesine rağmen EEG yöntemi kolay uygulanabilirlik, düşük maliyet, yüksek zaman çözünürlüğü, gerçek zamanlı çalışma imkânı sunması ve noninvazif olması avantajlarından dolayı tercih edilmektedir. Yavaş Kortikal Potansiyeller (SCP), P300, Uyarılmış Potansiyeller gibi farklı sinyaller ile BBA sistemleri tasarlanabilir. Sinyal kaynağı olarak durağan hal görsel uyarılmış potansiyel kullanılarak yüksek hızlı ve eğitim gerektirmeyen BBA sistemleri gerçekleştirilebilir $[5,6]$.

DHGUP sinyalleri $3,5 \mathrm{~Hz}-75 \mathrm{~Hz}$ frekanslarında yanıp sönen görsel uyaran sonucu oksipital bölgede ortaya çıkmaktadır. Bu sinyaller sinüzoidal dalga biçiminde sürekli ve periyodik bir sinyallerdir ve frekansları görsel uyaranın frekansı ve tam katları ile eşleşmektedir. DHGUP sinyallerinin davranışı EEG sinyalinin spektrumunda rahatça görünmektedir. Şekil 1a'da 7 Hz'lik görsel uyaran esnasında elde edilen EEG sinyalinin spektrumu sunulmuştur. Görsel uyaran frekansındaki ve harmoniklerindeki bileşenler açıkça görülmektedir. Şekil 1b'de ise görsel uyarana maruz bir denekten elde edilen güç spektrumunun kafa topografisi görülmektedir. Şekilden de görüldüğü gibi görsel uyaran oksipital bölge ve çevresinde güç artışına sebep olurken diğer alanlarda görsel uyaranın belirgin bir etkisi gözlemlenmemektedir.

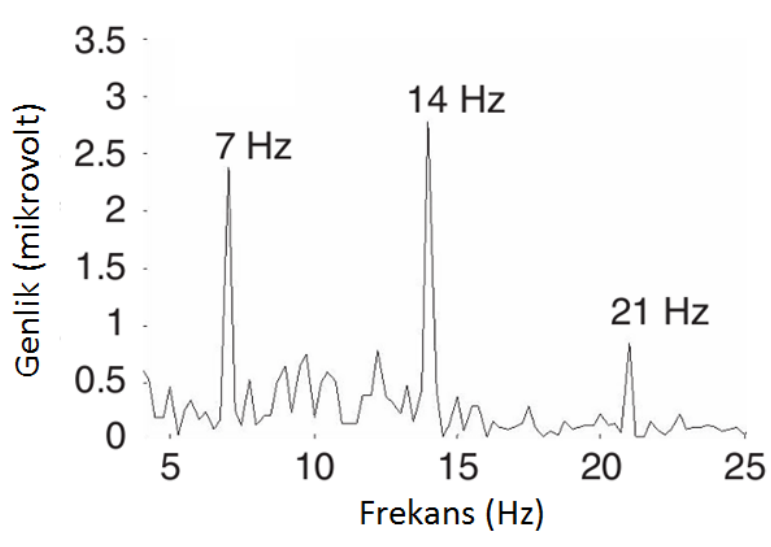

(a)

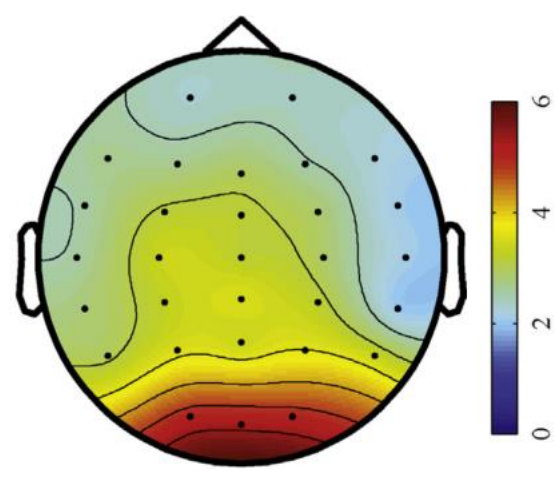

(b)

Şekil 1. a) 7 Hz'lik görsel uyaran esnasında elde edilen EEG sinyalinin spektrumu [7] b) Güç topografisi [8]

DHGUP sinyalleri ile BBA tasarımı için, kullanıcıya yanıp sönen led ışıklar veya monitörde yanıp sönen kutucuklar gibi görsel uyaranlar gösterilir. Bu görsel uyaranların tamamı frekanslarının farklı olması gibi ayırt edici özellikler taşımaktadır ve BBA sistemi çıkışındaki uygulamada farklı bir komut ile ilişkilidir. Kullanıcı görsel uyaranlardan birine odaklandığında, beyinde görme duyusuyla ilgili bilgilerin işlendiği oksipital bölgede, odaklanılan görsel uyaranın özelliklerini yansıtan DHGUP sinyali ortaya çıkmaktadır. Bu esnada kişiden alınan EEG sinyallerine uygulanan sinyal işleme metotları ile hedef görsel uyaran tespit edilmeye çalış1ır. Hedef görsel uyaranın tespit edilmesi ile sistem çıkışındaki ilişkili komut (fare imleci kontrolü...) icra edilir (Şekil 2) [9]. 


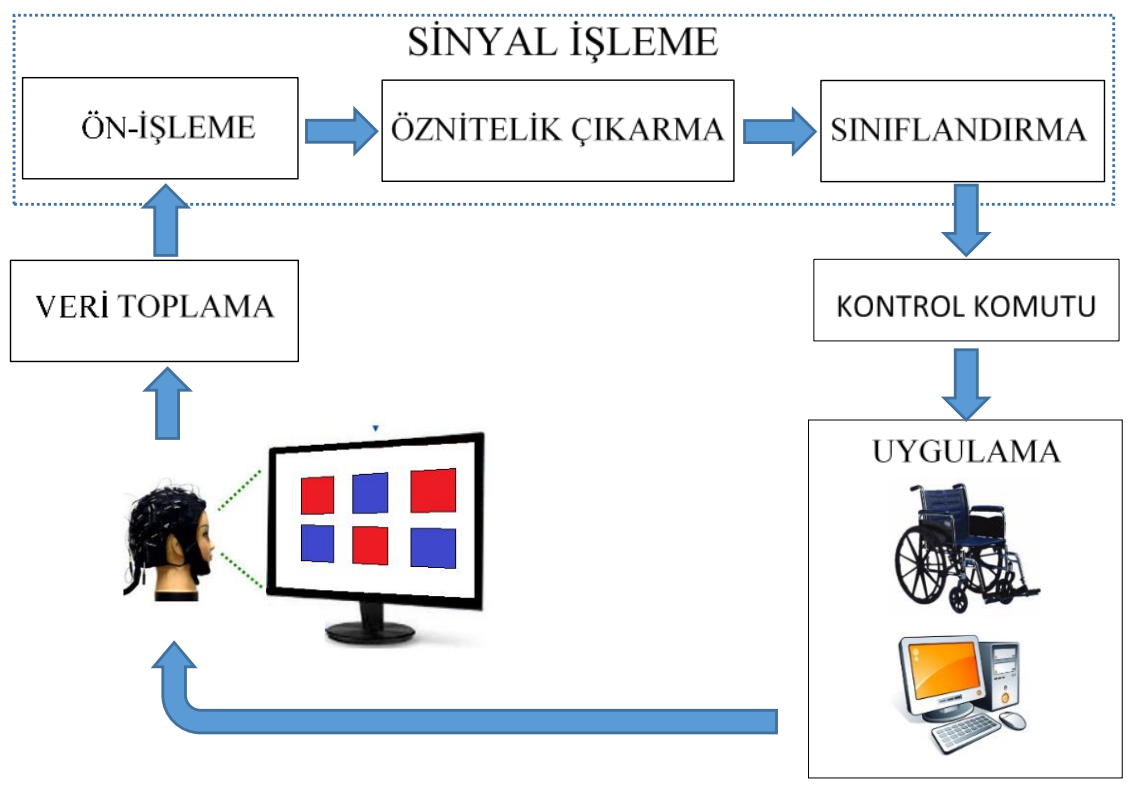

Şekil 2. DHGUP temelli BBA sisteminin genel yapısı [10]

$\mathrm{Bu}$ çalışma kapsamında pratik bir EEG cihazı olan Emotiv Epoc ile Matlab ortamında DHGUP tabanlı BBA tasarlanmış ve hayata geçirilmiştir. Görsel uyaran kaynağı olarak monitörün kullanıldığ1 bu çalışmaya 5 denek katılmıştır. Deneyler ön hazırlık ve gerçek zamanlı olarak iki kısımdan oluşmaktadır. Ön hazırlık aşaması ile kullanıcının sisteme alışması ve gerçek zamanlı uygulamada kullanılacak olan DHGUP tespit yöntemi ve tespit süresine karar verilmesi amaçlanmıştır. Farklı DHGUP tespit yöntemleri ile değişen sinyal sürelerinde elde edilen tespit doğruluğuna dayanarak gerçek zamanlı BBA uygulamasında kullanılacak yöntem ve süreye karar verilmiştir. Gerçek zamanlı uygulamada ise tuş takımı şeklinde tasarlanmış olan görsel uyaran düzeneği ile kullanıcıların yalnız monitördeki sayılara odaklanarak telefon numaralarını yazması istenmiştir.

Bu makalede 2. bölümde EEG sinyallerinin toplanması, kullanılan görsel uyaranlar ve DHGUP tespit yöntemleri açıklanmış, 3. bölümde ön hazırlık ve gerçek zamanlı deneylerin sonuçları sunulmuş ve yorumlanmıştır. 4 . bölümde ise sonuçlar ve önerilere yer verilmiştir.

\section{Materyal ve Metot}

\subsection{Veri Toplama ve Deney Düzeneği}

Tasarlanan DHGUP temelli BBA sisteminde EEG sinyallerinin elde edilmesinde Emotiv Epoc cihazı kullanılmıştır. Emotiv Epoc, taşınabilir, 2'si referans olmak üzere 16 kanallı, pille çalışan, sinyalleri bluetooth haberleşme yöntemi ile bilgisayara aktaran, ucuz, tüketici seviyesinde bir EEG cihazıdır. Cihazın kullanımı herhangi bir uzmanlık gerektirmemektedir. EEG ölçümü için jele ihtiyaç yoktur. Cihazın asıl hedefi oyun ve eğlence uygulamaları [11] olmasına rağmen BBA sistemlerinde [12] ve sinir - bilim gibi araştırma alanlarında da [13] kullanılmaktadır. Cihaz 14 bit çözünürlüğe, 128 örnek/saniye örnekleme frekansina sahiptir.

Cihazın bu artılarının yanında toplanan sinyallerin gürültülü olması ve sabit uzunluklu elektrot kolları (Şekil 3) DHGUP temelli BBA uygulamasını zorlaştırmaktadır. Cihazdaki 14 elektrottan yalnız ikisi oksipital bölge civarına düşmektedir. Ayrıca sabit uzunluklu elektrot kolları farklı boyutlardaki esnek EEG şapkalarının aksine ölçüm noktalarını kısıtlamaktadır. Farklı kafa boyutuna sahip deneklerde elektrotlar istenilen ölçüm noktalarına ulaşmamaktadır. Bu durum çalışmaları olumsuz etkilemektedir [14]. Bu olumsuzluğu kısmen azaltmak için cihaz 180 derece döndürülerek kullanılmıştır. Bu sayede AF3 F3 F4 AF4 konumları için tasarlanan 4 elektrot, oksipital bölge civarına gelmektedir. 

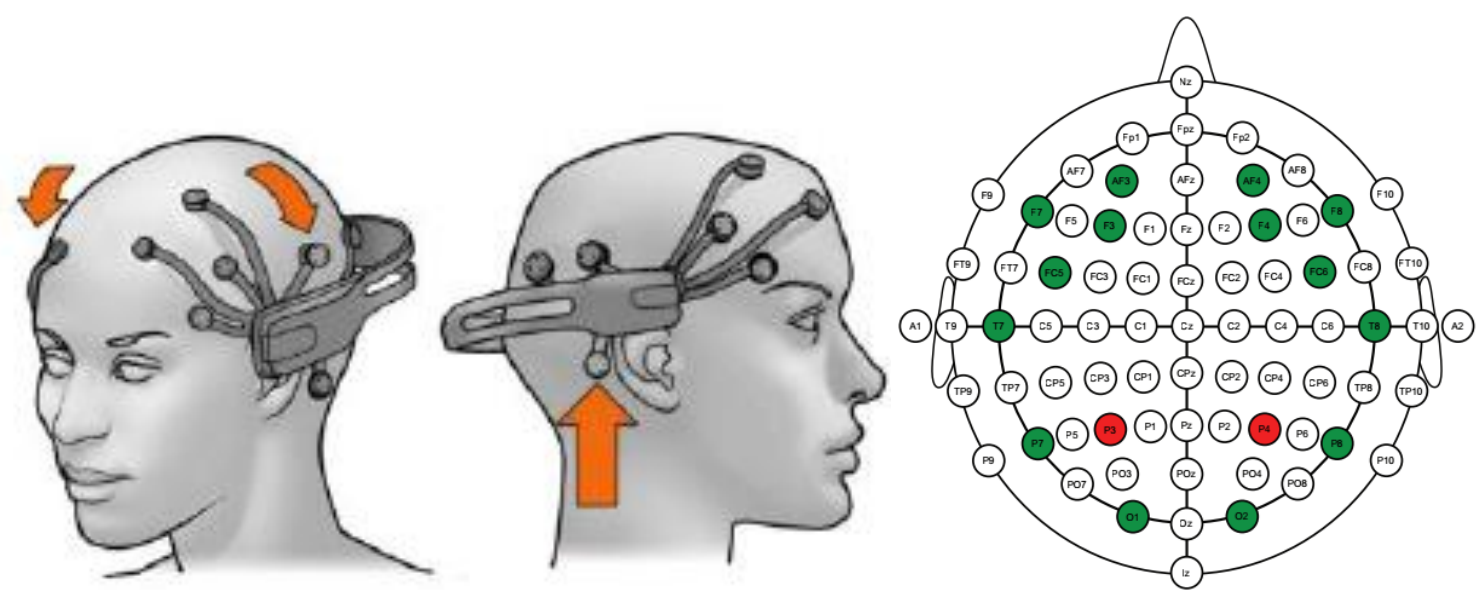

Şekil 3. Emotiv Epoc cihazı elektrot konumları. Yeşil kanallar ölçüm elektrotlarını kırmızı kanallar referans elektrotları göstermektedir

Çalışmada görsel uyaran kaynağı için monitör kullanılmıştır. Görsel uyaranlar Matlab ortamında "Cogent Graphics Toolbox" kullanılarak sağlanmıştır [15]. Ön hazırlık aşaması için Şekil 4a'da görülen tek bir görsel uyaran barındıran düzenek kullanılmıştır. Ön hazırlık deneyleri 10 oturumdan oluşmaktadır. Her bir oturumda denekler 12 adet denemeye katılmıştır. Denemelerde uyaran sirasıly $6.33,5.45,6,6.66,7,7.5,8,8.57,9,9.5,10.5$ ve $11.5 \mathrm{~Hz}$ hizlarında yanıp sönmektedir. Denekler her bir denemede 5 saniye boyunca uyarana odaklanmıştır. Denemeler arası dinlenme süresi 1,5 saniyedir. Oturumlar arasındaki dinlenme süresi ise deneğe bırakılmıştır.

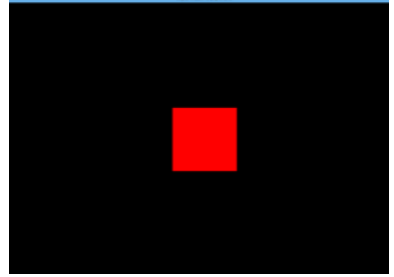

(a)

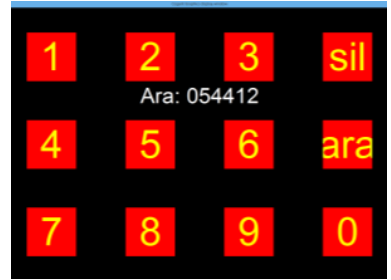

(b)

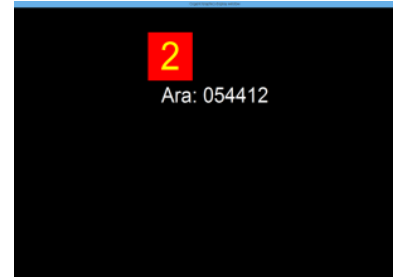

(c)

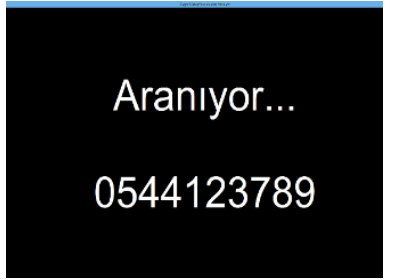

(d)

Şekil 4. Görsel uyaran düzeneği

Gerçek zamanlı deneyler için ise Şekil 4b'de görülen 6.33, 5.45, 6, 6.66, 7, 7.5, 8, 8.57, 9, 9.5, 10.5 ve $11.5 \mathrm{~Hz}$ frekanslarına sahip 12 uyarandan oluşan düzenek tasarlanmıştır. Şekil 4b'de görüldüğü gibi görsel uyaran düzeneği tuş seti şeklindedir. Gerçek zamanlı deneylerde kullanıcılardan istedikleri bir telefon numarasını BBA aracılığı ile yazdırmaları istenmiştir. Kullanıcı belirlenen tespit süresince uyaranlardan birine odaklanır. Ardından bu süre zarfında toplanan EEG sinyallerinden DHGUP tespiti yapılır ve ekranda 0,5 saniye süresince yalnızca tespit edilen uyaran görünmektedir (Şekil 4c). Denekler "sil" isimli uyaran ile son yazdırdı̆̆ numarayı silebilir. "ara" isimli görsel uyarana odaklanarak deneyi sonlandırabilir (Şekil 4d).

\subsection{Güç Spektral Yoğunluğu Analizi ile DHGUP Tespiti}

DHGUP sinyali görsel uyaranın frekansında sinüzoidal bir sinyal olduğu için EEG sinyalinin spektrumu incelenerek DHGUP tespiti yapılabilir. Güç spektral yoğunluğu analizi (GSYA) ile EEG sinyalinin görsel uyaran frekanslarındaki bileşenlerinin güç değerleri elde edilir. Elde edilen güç değerleri karşılaştırılarak DHGUP tespiti yapılmaktadır.

$f=\operatorname{argmax}_{i} P\left(f_{i}\right), \quad i=1,2, \ldots, K$

Denklem 1'de $K$ görsel uyaran sayısı, $P\left(f_{i}\right) f_{i}$ frekansındaki güç değeri ve $f$ odaklanılan uyaranın frekansıdır. Spektrumun elde edilmesinde çoğunlukla Hızlı Fourier Dönüşümü kullanılmaktadır [16, 17]. 


\subsection{Kanonik Korelasyon Analizi ile DHGUP Tespiti}

DHGUP temelli BBA sistemlerinde çoklu elektrot sinyallerinden hedef uyaran tespiti Kanonik korelasyon analizi (KKA) ile yapılabilir $[18,19]$. Çok boyutlu iki veri seti arasındaki ilişki KKA yöntemi ile tespit edilebilir. KKA'da 2 veri setinin $(X, Y)$ koordinat sistemlerini en ilişkili hale getirecek ağırlık vektörleri $\left(w_{x}, w_{y}\right)$ hesaplanır. Veri setleri $(X, Y)$ ve hesaplanan ağıllık vektörlerinin lineer kombinasyonuyla $\left(X^{T} w_{x}, Y^{T} w_{y}\right)$ kanonik değişkenler $\left(x=X^{T} w_{x}, y=Y^{T} w_{y}\right)$ elde edilir. Bu kanonik değişkenler arasındaki korelasyon değeri kanonik korelasyon katsayısı olarak adlandırılır. Kanonik korelasyon katsayısı denklem 2 ile elde edilmektedir.

$$
\max _{w_{x} w_{y}} \rho(x, y)=\frac{E\left[x y^{T}\right]}{\sqrt{E\left[x x^{t}\right] E\left[y y^{T}\right]}}=\frac{E\left[w_{x}^{T} X Y^{T} w_{y}\right]}{\sqrt{E\left[w_{x}^{T} X X^{T} w_{x}\right] E\left[w_{y}^{T} Y Y^{T} w_{y}\right]}}
$$

Denklem 2'de $\rho(x, y)$ kanonik değişkenler arasındaki korelasyon katsayısıdır. DHGUP temelli BBA sistemlerinde KKA yöntemi ile çoklu kanal EEG sinyalleri ve referans sinyaller arasındaki ilişki incelenmektedir. Referans sinyaller uyaran frekansında ve harmoniklerinde sinüs ve kosinüs fonksiyonları ile oluşturulmaktadır (Denklem 3).

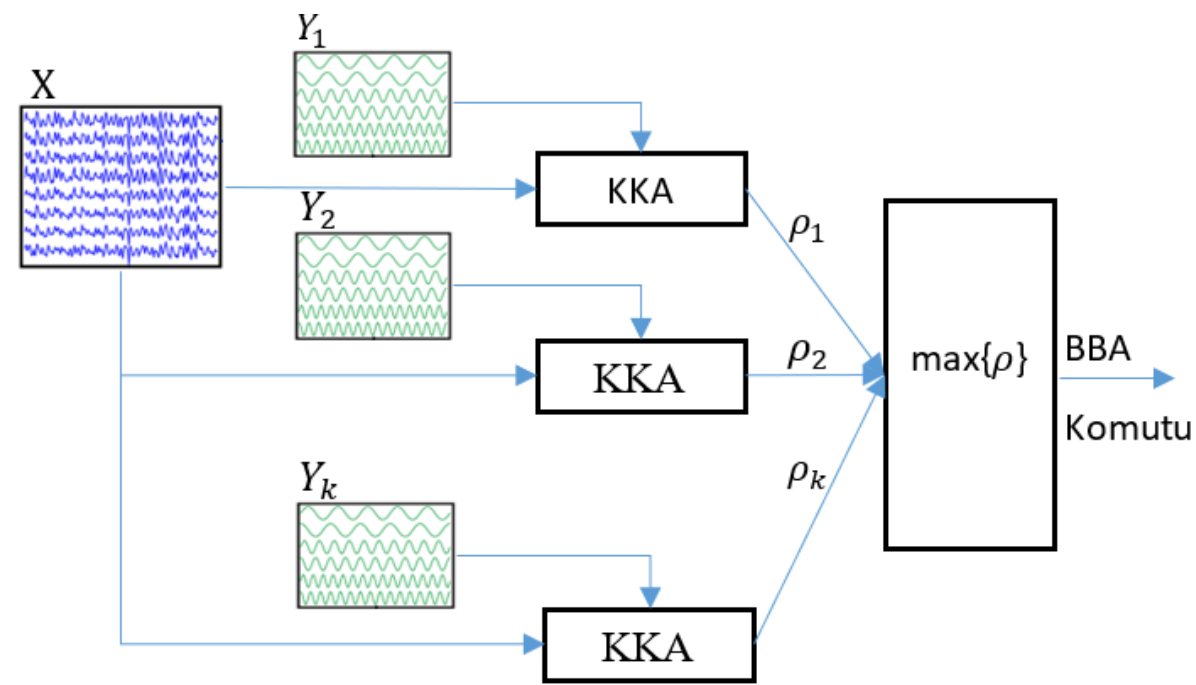

Şekil 5. KKA ile DHGUP tespiti

$Y_{i}=\left[\begin{array}{c}\sin \left(2 \pi f_{i} t\right) \\ \cos \left(2 \pi f_{i} t\right) \\ \vdots \\ \sin \left(2 \pi N_{h} f_{i} t\right) \\ \cos \left(2 \pi N_{h} f_{i} t\right)\end{array}\right], t=\left[\frac{1}{f_{s}}, \frac{2}{f_{s}}, \ldots, \frac{N_{s}}{f_{s}}\right]$

Burada $f_{i}$ uyaran frekansını $N_{h}$ harmonik numarasını göstermektedir. Şekil 5'de KKA yönteminin işleyişi görülmektedir. Uyaran frekanslarındaki referans sinyaller ve çoklu kanal EEG sinyalleri ile elde edilen kanonik korelasyon katsayıları karşılaştırılarak DHGUP tespiti yapılmaktadır (Denklem 4).

$f=\operatorname{argmax}_{i} \rho\left(f_{i}\right), \quad i=1,2, \ldots, K$

\subsection{Bireysel Şablon Destekli Kanonik Korelasyon Analizi ile DHGUP Tespiti}

KKA metodu kolay uygulanabilir olması ve kısa süreli EEG sinyallerinde dahi doğru DHGUP tespiti sağlamasından dolayı farklı yaklaşımlar ile geliştirilmektedir [20,21]. Bireysel şablon destekli kanonik 
korelasyon analizinde (BŞDKKA) sinüzoidal referans sinyallerine ek olarak ön hazırlık aşaması ile elde edilen deneklerin ortalama EEG sinyali de işleme dahil edilmektedir. Şekil 6'da görüldüğü gibi, KKA yöntemi ile referans sinyaller $(Y)$, test sinyalleri $(X)$ ve bireysel şablon sinyallerden $(\hat{X})$ ağırlık katsayıları hesaplanmıştır $\left(W_{X \hat{X}}, W_{X Y}, W_{\hat{X} Y}\right) .2$. adımda bu ağırlık katsayıları ile bireysel şablon sinyalleri ve test sinyalleri çarpılmaktadır. Son adımda çarpım sonucu elde edilen sinyallerin korelasyon değerlerinin toplamı $(\tilde{\rho})$ kullanılarak DHGUP tespiti yapılmaktadır.

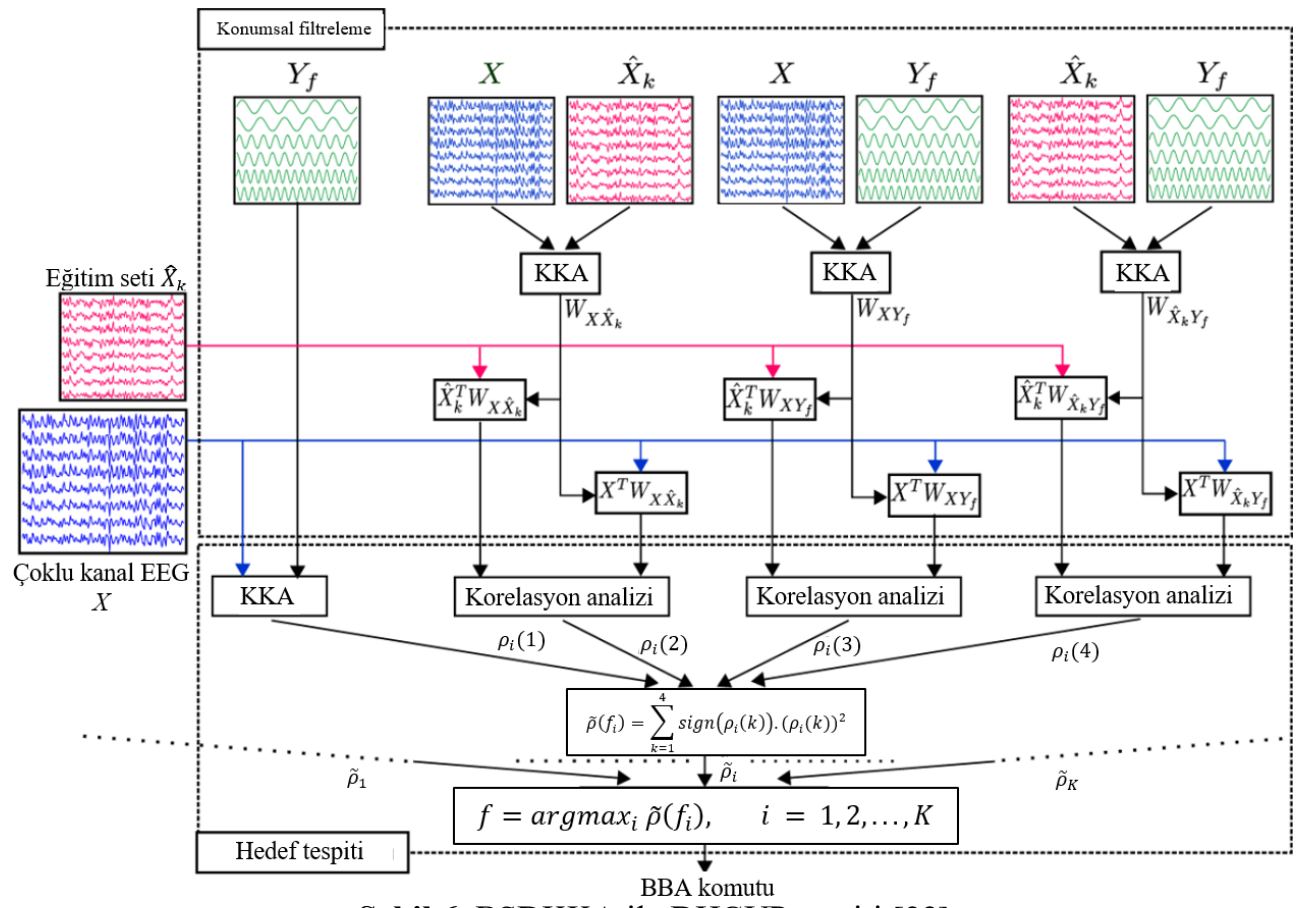

Şekil 6. BŞDKKA ile DHGUP tespiti [22]

Yöntemin matematiksel ifadesi denklem 5 ve 6 'daki gibidir.

$\boldsymbol{\rho}_{i}=\left[\begin{array}{c}\rho_{i}(1) \\ \rho_{i}(2) \\ \rho_{i}(3) \\ \rho_{i}(4)\end{array}\right]=\left[\begin{array}{c}\rho(X, Y) \\ \rho\left(X^{T} W_{X \hat{X}}, \hat{X}^{T} W_{X \hat{X}}\right) \\ \rho\left(X^{T} W_{X Y}, \hat{X}^{T} W_{X Y}\right) \\ \rho\left(X^{T} W_{\hat{X} Y}, \hat{X}^{T} W_{\hat{X} Y}\right)\end{array}\right] i=1,2, \ldots, K$
$\tilde{\rho}\left(f_{i}\right)=\sum_{k=1}^{4} \operatorname{sign}\left(\rho_{i}(k)\right) \cdot\left(\rho_{i}(k)\right)^{2} \quad i=1,2, \ldots, K$

Burada $X$ test sinyalleri, $Y$ referans sinyaller $\hat{X}$ bireysel şablonlar ve $\rho$ korelasyon katsayısıdır. Elde edilen toplam korelasyon katsayıları karşılaştırılarak DHGUP tespiti yapılmaktadır (Denklem 7).

$f=\operatorname{argmax}_{i} \tilde{\rho}\left(f_{i}\right), \quad i=1,2, \ldots, K$

\section{Bulgular ve Tartışma}

DHGUP tabanlı BBA sisteminde kullanılacak EEG kayıt süresine ve DHGUP tespit yöntemine karar verilmesi için ön hazırlık deneyleri ile EEG sinyalleri kaydedilmiş ve analiz edilmiştir. Materyal ve Metot bölümünde bahsedildiği gibi, her bir denek için 4 kanaldan her biri 12 adet denemeden oluşan 10 oturum sonucu 480 adet 5 saniyelik EEG sinyalleri elde edilmiştir. Sinyaller $5-23 \mathrm{~Hz}$ sonsuz dürtü yanıtlı (IIR) bant geçiren fitre ile filtrelenmiştir. Filtrenin sınırları görsel uyaran frekanslarının ilk ve ikinci harmoniğini kapsayacak şekilde seçilmiştir. DHGUP sinyali, göz veya vücut hareketi kaynaklı gürültülere karşı bağışıklığı olduğu [23] için çalışmada bant geçiren filtrenin dişında ön-işleme uygulanmamıştır. Filtrelenmiş sinyallerden KKA, BŞDKKA ve GSYA yöntemleri ile DHGUP tespiti yapılmıştır. GSYA analizinde 4 kanaldan alınan sinyal ayrı ayrı değerlendirilirken, çoklu kanal yöntemi olan KKA ve BŞDKKA için 4 kanaldan alınan sinyaller beraber değerlendirilmiştir. GSYA analizinin 
4 kanala ayrı ayrı uygulanması ile deneklerdeki DHGUP sinyalinin en yoğun gözlemlendiği kanal tespit edilmiştir.

5 denekten değişen sinyal uzunluklarında elde edilen tespit doğruluğu ve ortalama değerler Şekil 7'de görülmektedir.

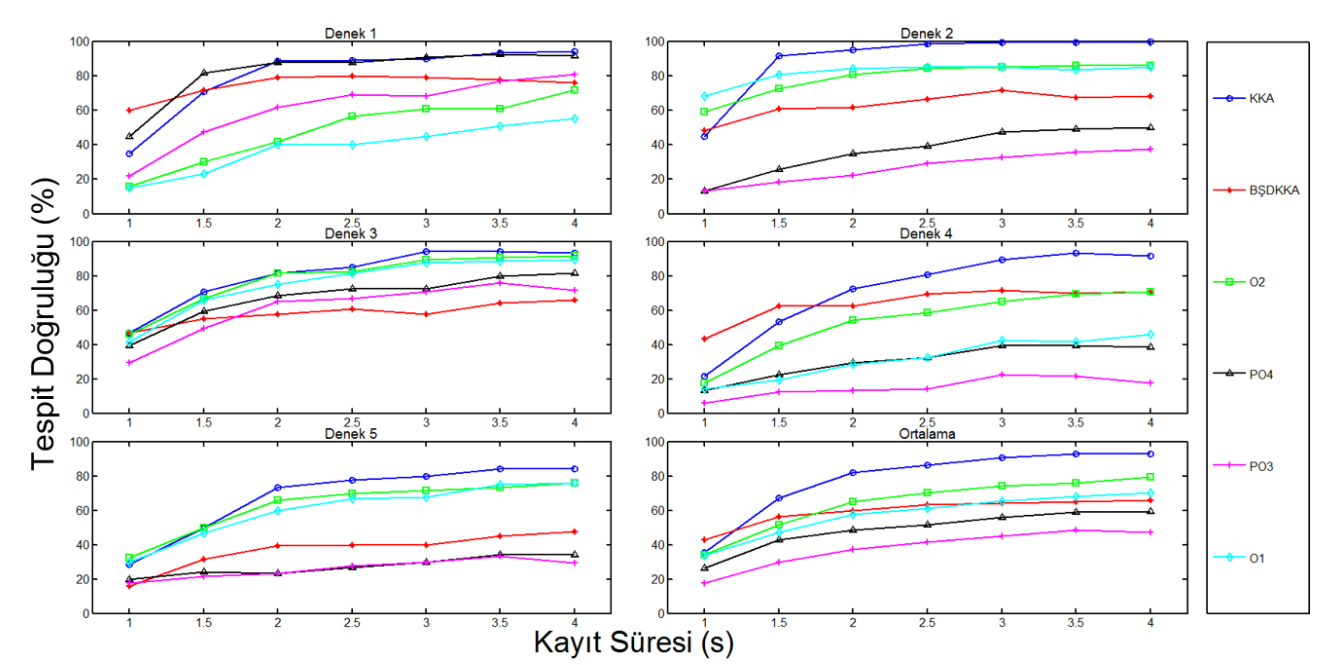

Şekil 7. Ön hazırlık aşamasında elde edilen tespit doğruluğu

Şekil 7'de O1 O2 PO4 PO3 etiketleri ile bu kanallardan GSYA ile elde edilen tespit doğruluğu sunulmuştur. Ön hazırlık deneyleri sonuçları incelendiğinde KKA yöntemi ile daha yüksek tespit doğruluğu elde edildiği görülmektedir. Bundan dolayı gerçek zamanlı uygulamada KKA yöntemi tercih edilmiştir.

Çalışmalarda $[5,22,24]$ BŞDKKA ile yüksek tespit doğruluğu elde edilmiş olmasına rağmen ön hazırlık deneylerinde sonuçların tatmin edici olmamasının sebebi bireysel şablonun tutarsızlığı olabilir. Şablon, aynı frekanstaki DHGUP bileşenine sahip sinyallerin ortalaması alınarak elde edilmektedir. Kullanışı bir şablon elde edilebilmesi için DHGUP bileşenlerinin faz değerlerinin benzer olması gerekmektedir. Bu ise EEG kayıt sistemi ile görsel uyaran sisteminin eş zamanlı çalışması ile mümkün olmaktadır. Emotiv Epoc cihazındaki kısıtlamalar ve Matlab yazılımından kaynaklanan gecikmeler ise kullanılabilir bir şablona ulaşılmasını zorlaştırmaktadır.

Gerçek zamanlı deneylerde kullanılan kayıt süresi, bilgi aktarım hızı (BAH) değerlendirilerek belirlenmiştir. BAH birim zamanda iletilen bilgi miktarıdır. BAH terimi, BBA sistemindeki seçenek sayısına, tespit süresi ve doğruluğuna bağlıdır.

$B A H=\frac{60}{T} \times\left[\log _{2} N+P \log _{2} P+(1-P) \log _{2} \frac{(1-P)}{(N-1)}\right]$

Burada $T$ tespit süresini, $P$ tespit doğruluğunu $(0-1$ arası), $N$ seçenek sayısını ifade etmektedir. Ön hazırlık deneylerinde KKA yöntemi ile farklı tespit sürelerinde elde edilen BAH değerleri Tablo 1'de sunulmuştur.

Tablo 1. KKA ile elde edilen tespit doğruluğu ve BAH değerleri

\begin{tabular}{|l|l|l|l|l|l|l|l|l|l|l|}
\hline Süre (sn.) & 0.5 & 1 & 1.5 & 2 & 2.5 & 3 & 3.5 & 4 & 4.5 & 5 \\
\hline Doğruluk (\%) & 13.3 & 35.3 & 67.3 & 82.2 & 86.2 & 90.5 & 92.8 & 92.6 & 92.6 & 92.6 \\
\hline BAH & 2.4 & 24.6 & 61.7 & 68.8 & 60.7 & 56.1 & 50.8 & 44.2 & 39.3 & 35.4 \\
\hline
\end{tabular}

Tablo 1'e göre gerçek zamanlı uygulama için yüksek BAH sağlayan tespit süresi 2 saniyedir. Bu sebeple gerçek zamanlı uygulamada 2 saniyelik EEG sinyallerinden DHGUP tespiti yapılmıştır. Tespit sonrası ekranda 0,5 saniye tespit edilen uyaran gösterildiği için kullanıc1 2,5 saniyede bir numara yazdırabilmektedir. Böylece tasarlanan BBA 24 karakter/dakika hızında çalışmaktadır. Gerçek zamanlı deneyler sonucu elde edilen sonuçlar Tablo 2'de görülmektedir. Sonuçlar incelendiğinde 1. ve 2. 
deneğin hatasız olarak hedefteki numaraya ulaştı̆̆ görülmektedir. 3 . denekte $2 \mathrm{kez}$, 5 . denekte 3 kez ve 4. denekte 5 kez hedef doğru tespit edilememiştir.

Tablo 2. Gerçek zamanlı uygulama sonuçları $(\sqrt{ }:$ ara, $\leftarrow$ :sil $)$.

\begin{tabular}{|l|l|l|c|}
\hline Denekler & Hedef & Tespit Edilen & Hedef/Tespit Sayıs \\
\hline Denek 1 & $05444637355 \checkmark$ & $05444637355 \checkmark$ & $12 / 12$ \\
\hline Denek 2 & $05056324751 \checkmark$ & $05056324751 \checkmark$ & $12 / 12$ \\
\hline Denek 3 & $05533144941 \checkmark$ & $0554 \longleftarrow 335 \leftarrow 144941 \checkmark$ & $12 / 16$ \\
\hline Denek 4 & $05075893657 \checkmark$ & $03 \leftarrow 507585 \longleftarrow 936 \leftarrow \leftarrow 368 \leftarrow 57 \checkmark$ & $12 / 22$ \\
\hline Denek 5 & $05075891415 \checkmark$ & $0507 \leftarrow 7581 \leftarrow 914 \leftarrow 415 \checkmark$ & $12 / 18$ \\
\hline
\end{tabular}

Gerçek zamanlı deneylerden elden edilen sonuçlara göre tasarlanan BBA ile kullanıcılar 11 haneli telefon numarasını ortalama 40 saniyede seçebilmiştir.

Deneysel sonuçlarda gösterildiği gibi Emotiv Epoc ile BBA tasarlanmış olmasına rağmen cihazın oksipital bölgede yalnızca 2 elektrotu olması, sabit uzunluklu elektrot kolları sebebiyle elektrotların istenen bölgeye ulaşmaması, görsel uyaran ile eş zamanlı çalışmanın zorluğu etkenlerinden dolayı DHGUP temelli BBA sistemleri için tam uygun olmadığı gözlemlenmiş̧ir.

\section{Sonuç ve Öneriler}

$\mathrm{Bu}$ çalışmada pratik ve düşük maliyetli bir EEG cihazı olan Emotiv Epoc ile DHGUP temelli BBA tasarlanmış ve hayata geçirilmiştir. Tasarlanan BBA tuş takımı biçiminde tasarlanan görsel uyaran düzeneği sayesinde kullanıcıların yalnızca beyin sinyalleri ile telefon numarası yazmalarına olanak sağlamaktadır. 5 kullanıcı ile yapılan deneylerde sistemin ortalama 40 saniyede 11 haneli bir telefon numarasını yazdırabildiği gösterilmiştir. Ayrıca çalışmada yüksek DHGUP tespit doğruluğu sağlayan eğitim verisi destekli BŞDKKA yönteminin Emotiv Epoc ile uygulanabilirliği incelenmiştir. Yöntem, daha önce bahsedilen eş zamanlı çalışma zorluğundan dolayı yaygın yöntemler olan GSY ve KKA'ya göre daha yüksek DHGUP tespiti sağlamamıştır. Görsel uyaran ile EEG cihazı arasındaki eş zamanlılık problemini aşmak için Matlab yerine farklı bir platform veya Matlab ortamında farklı bir toolbox tercih edilebilir. Bununla birlikte kullanılan cihaz, görsel uyaran tasarımı ve tespit yöntemi hakkında genel bir kanıya varmak için daha yüksek sayıda kullanıcı ile deneylerin yapılması gerekmektedir.

\section{Teşekkür}

Bu çalışma Karabük Üniversitesi Bilimsel Araştırma Projesi kapsamında desteklenmiştir (Proje No: KBÜ-BAP-14/2-DR-011).

\section{Kaynaklar}

[1] Kokswijk J.V., Hulle M.V. 2010. Self adaptive BCI as service-oriented information system for patients with communication disabilities, 2010 4th International Conference on New Trends in Information Science and Service Science (NISS), 264-269, 11-13 May 2010, Gyeongju.

[2] Santhosh J., Bhatia M., Sahu S., Anand S. 2004. Quantitative EEG analysis for assessment to "plan" a task in amyotrophic lateral sclerosis patients: a study of executive functions (planning) in ALS patients. Brain Res Cogn Brain Res, 22 (1): 59-66.

[3] Wolpaw J.R., Birbaumer N., McFarland D.J., Pfurtscheller G., Vaughan T.M. 2002. Braincomputer interfaces for communication and control. Clinical Neurophysiology, 113 (6): 767-791. doi:10.1016/S1388-2457(02)00057-3

[4] Sellers E.W., Vaughan T.M., Wolpaw J.R. 2010. A brain-computer interface for long-term independent home use. Amyotrophic Lateral Sclerosis, 11 (5): 449-455.

[5] Chen X., Wang Y., Nakanishi M., Gao X., Jung T.P., Gao S. 2015. High-speed spelling with a noninvasive brain-computer interface. Proceedings of the National Academy of Sciences, 112 (44): 1-10. doi:10.1073/pnas.1508080112

[6] Sözer A.T., Fidan C.B. 2017. Novel Detection Features for SSVEP Based BCI: Coefficient of 
Variation and Variation Speed. BRAIN: Broad Research in Artificial Intelligence and Neuroscience, 8 (2): 144-150.

[7] Wang Y., Gao X., Hong B., Jia C., Gao S. 2008. Brain-Computer Interfaces Based on Visual Evoked Potentials. IEEE Engineering in Medicine and Biology Magazine, 27 (5): 64-71.

[8] Zhang Y., Zhou G., Jin J., Wang X., Cichocki A. 2015. SSVEP recognition using common feature analysis in brain-computer interface. Journal of Neuroscience Methods, 244: 8-15.

[9] Sozer A.T., Fidan C.B. 2016. Implementation of a steady state visual evoked potantial based brain computer interface. In 2016 24th Signal Processing and Communication Application Conference (SIU) (pp. 1173-1176). IEEE. doi:10.1109/SIU.2016.7495954

[10] Sözer A.T., Fidan C.B. 2018. Novel spatial filter for SSVEP-based BCI: A generated reference filter approach. Computers in Biology and Medicine, 96: 98-105.

[11] Van Vliet M., Robben A., Chumerin N., Manyakov N.V., Combaz A., Van Hulle M.M. 2012. Designing a brain-computer interface controlled video-game using consumer grade EEG hardware. In 2012 ISSNIP Biosignals and Biorobotics Conference: Biosignals and Robotics for Better and Safer Living, BRC 2012 (pp. 1-6). IEEE. doi:10.1109/BRC.2012.6222186

[13] Choi B., Jo S. 2013. A Low-Cost EEG System-Based Hybrid Brain-Computer Interface for Humanoid Robot Navigation and Recognition. PLoS ONE, 8(9): e74583. doi:10.1371/journal.pone.0074583.

[14] Badcock N.A., Mousikou P., Mahajan Y., de Lissa P., Thie J., McArthur G. 2013. Validation of the Emotiv EPOC ${ }^{\circledR}$ EEG gaming system for measuring research quality auditory ERPs. PeerJ, 1 (1): e38. doi:10.7717/peerj.38.

[15] Chumerin N., Manyakov N.V., Van Vliet M., Robben A., Combaz A., Van Hulle M.M. 2013. Steady-state visual evoked potential-based computer gaming on a consumer-grade EEG device. IEEE Transactions on Computational Intelligence and AI in Games, 5(2): 100-110.

[16] Cogent Graphics. 2017. Laboratory of Neurobiology. Retrieved July 1, 2017, from http://www.vislab.ucl.ac.uk/cogent_graphics.php

[17] Hwang H.J., Lim J.H., Jung Y.J., Choi H., Lee S.W., Im C.H. 2012. Development of an SSVEPbased BCI spelling system adopting a QWERTY-style LED keyboard. Journal of Neuroscience Methods, 208 (1): 59-65. doi:10.1016/j.jneumeth.2012.04.011

[18] Sanchez G., Diez P.F., Avila E., Leber E.L. 2011. Simple communication using a SSVEP-based BCI. Journal of Physics: Conference Series, 332 (1): 012017. doi:10.1088/17426596/332/1/012017.

[19] Yan Z., Gao X., Bin G., Hong B., Gao S. 2009. A half-field stimulation pattern for SSVEP-based brain-computer interface. Conference proceedings : ... Annual International Conference of the IEEE Engineering in Medicine and Biology Society. IEEE Engineering in Medicine and Biology Society. Annual Conference, 2009, 6461-4. doi:10.1109/IEMBS.2009.5333544.

[20] Lin Z., Zhang C., Wu W., Gao X. (007) Frequency recognition based on canonical correlation analysis for SSVEP-Based BCIs. IEEE Transactions on Biomedical Engineering, 54 (6): 11721176. doi:10.1109/TBME.2006.889197.

[21] Poryzala P., Materka A. 2014. Cluster analysis of CCA coefficients for robust detection of the asynchronous SSVEPs in brain-computer interfaces. Biomedical Signal Processing and Control, 10 (1): 201-208.

[22] Cao L., Ju Z., Li J., Jian R., Jiang C. 2015. Sequence detection analysis based on canonical correlation for steady-state visual evoked potential brain computer interfaces. Journal of Neuroscience Methods, 253: 10-17.

[23] Nakanishi M., Wang Y., Wang Y.T., Mitsukara Y., Jung T.P. 2014. A High-Speed Brain Speller Using Steady-State Visual Evoked Potentials. International Journal of Neural Systems, 24 (06): 1450019. doi:10.1142/S0129065714500191.

[24]Wu Z., Su S. 2014. A dynamic selection method for reference electrode in SSVEP-based BCI. PLoS ONE, 9 (8): e104248. doi:10.1371/journal.pone.0104248.

[25] Nakanishi M., Wang Y., Wang Y.T., Jung T.P. 2015. A Comparison Study of Canonical Correlation Analysis Based Methods for Detecting Steady-State Visual Evoked Potentials. PloS one, 10(10), e0140703. doi:10.1371/journal.pone.0140703 